\title{
Evaluation of wound healing process based on texture image analysis
}

\author{
Christos P. Loizou ${ }^{1,2}$, Takis Kasparis ${ }^{2}$, Michalis Polyviou ${ }^{3}$ \\ 1. Department of Computer Science, Intercollege, Limassol, Cyprus. 2. Department of Electrical Engineering, Computer \\ Engineering \& Informatics, Cyprus University of Technology, Limassol, Cyprus. 3. Polyviou Clinic, Limassol, Cyprus \\ Correspondence: Christos P. Loizou. Address: Intercollege, Dept. of Computer Science, School of Sciences and \\ Engineering, 92 Ayias Phylaxeos Str., P.O. Box 51604, CY-3507 Limassol, Cyprus. Email: christos.loizou@cut.ac.cy.
}

Received: October 20, 2012

Accepted: November 18, 2012 Online Published: March 28, 2013

DOI : $10.5430 /$ jbgc.v3n3p1

URL: http://dx.doi.org/10.5430/jbgc.v3n3p1

\begin{abstract}
Wound healing rate remains an interesting and important issue, in which modern imaging techniques have not yet given a definitive answer. In order to guide better therapeutic interventions, a better understanding of the fundamental mechanisms driving tissue repair are required. The wound healing rate is primarily quantified by the rate of change of the wound's surface area. The objective of this work is to establish a standardized and objective technique to assess the progress of wound healing in wounds appearing on patient's feet, by means of texture image analysis. Image pre-processing, segmentation, texture and geometrical analysis together with visual expert's evaluation were used to assess the wound healing process. A total of 77 digital images from 11 different subjects with foot wounds were taken every third day, for 21 days, by an inexpensive digital camera under different lighting conditions. The images were intensity normalized, and wounds were automatic segmented using a segmentation system based on snakes. From the segmented wounds, 56 different texture features and 4 different geometrical measures were extracted in order to identify features that quantify the rate of wound healing. Texture features that may indicate the progression of wound healing process were identified. More specifically, certain texture features increase (mean, contrast, roughness and radial sum), while some other texture features decrease (sum of squares variance, sum variance, sum average, entropy, coarseness, EE-laws texture energy measures and the Hurst coefficients for fractal dimension one and two analysis) with the progression of the wound healing process. These features were found to be significantly different at an observed time point during the wound healing process, when compared to previous different time points, and this could be used to indicate the rate of wound healing. No significant differences were found for all geometrical measures extracted from the wounds between different time points. Based on the results of this study, it is suggested that some texture features might be used to monitor the wound healing process, thus reducing the workload of experts, provide standardization, reduce costs, and improve the treatment quality for patients. The simplicity of the method also suggests that it may be a valuable tool in clinical wound evaluation. A larger scale study is needed to establish the application in clinical practice and for computing texture features and geometrical measures that may provide information for better and earlier differentiation of the wound healing process. Future work will incorporate additional texture features and geometrical measures for assessing the wound healing process in order to be used in the real clinical practice.
\end{abstract}

\section{Key words}

Wound segmentation, Texture analysis, Wound healing rate 


\section{I ntroduction}

Chronic wounds present an increasing health challenge as population ages and the incidence of different chronic diseases grows worldwide ${ }^{[1]}$. The progress of wound healing may be quantified by the rate of change of the wound's surface area ${ }^{[2]}$. However, this is a challenging task due to the complexity of the wound, variable lighting conditions, and the time constraints in clinical laboratories. A color image of a wound on the foot of a patient is presented in Figure 1a). One way to evaluate the wound healing rate is to monitor the wound status by acquiring images at regular patient visits (see Figure 2). If the physical dimensions of the wound are assessed at regular time intervals, then the experts will know if the patient is responding well or not to a particular treatment and if necessary re-adjust it ${ }^{[2]}$. In 2012 the 22nd annual meeting of the Wound Healing Society (WHS), set the standards for wound healing procedures and proposed recommendations for evaluating optimal wound treatments ${ }^{[3]}$.

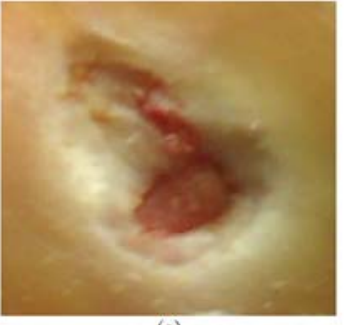

(a)

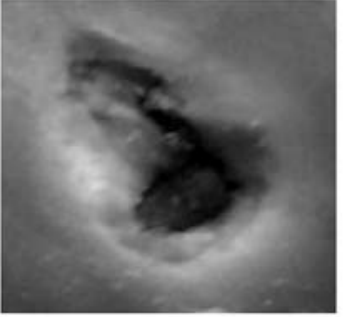

(c)

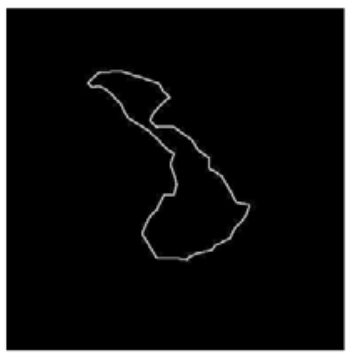

(e)

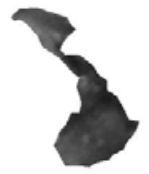

(g)

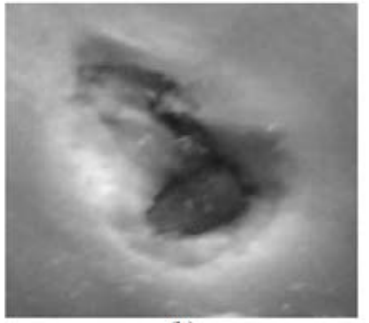

(b)

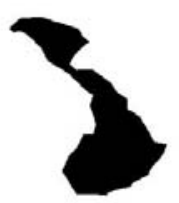

(d)

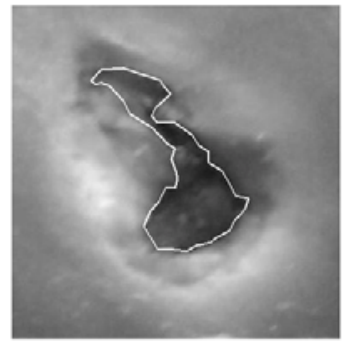

(f)

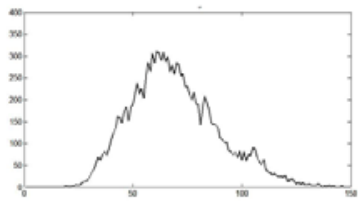

(h)

Figure 1. a) Original RGB color image of a wound on foot, from a patient at first visit, b) gray scale image of the wound, c) normalized image of the wound, d) binarised image of the wound, e) contour matrix of the binary wound image, f) final segmentation of the wound by snakes, g) segmented wound, h) histogram of the segmented wound. 
There are not many research groups worldwide that are involved in color image processing of wounds. Papazoglou et al. ${ }^{[2]}$ proposed and evaluated a highly accurate algorithm for wound segmentation which requires a minimal manual input by using a combination of both red-green-blue (RGB) and $\mathrm{L} * \mathrm{a} * \mathrm{~b} *$ color spaces, as well as a combination of threshold and pixel-based color comparing segmentation methods. Jones et al. ${ }^{[4]}$, and Jones ${ }^{[5]}$, developed the MAVIS system which is able to automatically measure the dimensions of skin wounds. Their method was based on color segmentation algorithms and was able to segment an image into one of three tissue types: healthy skin, wound tissue and epithelialisation tissue. Furthermore, six measurement parameters: the R, G and B color planes, hue, saturation and gray-level intensity were taken into consideration. The R, G and B color planes were only examined, in isolation showing that straightforward thresholding of color planes cannot produce a good segmentation, which distinguishes between wound and skin tissues. They found that wound segmentation is only partially succeeded if only the one-dimensional color histograms were taken into consideration, while using a 3-dimesional (3D) RGB histogram space, the color volume clusters may be more widely separated and a better segmentation result can be achieved. Mekkes et al. ${ }^{[6]}$ made some progress with such the 3D RGB color histogram clustering technique to assess the healing of wounds. It was shown that clusters in RGB space for a given tissue type formed an irregularly shaped 3D cloud, and therefore simple thresholding along the R, G and B axes would not help to segment the image into these three tissue types. Some other researchers presented their techniques on the segmentation of wounds in color images based on the use of the black-yellow-red classification scheme to evaluate the debridement activity of wounds ${ }^{[7]}$. A method to correct for limb convexity in color video images in order to measure the size of skin wounds and ulceration was presented ${ }^{[8]}$, while techniques to evaluate wound repair in humans and animals using basic color image processing were proposed in ${ }^{[9]}$. In another study ${ }^{[10]}$, a Support Vector Machine classifier (SVM) ${ }^{[11]}$, was employed to perform region segmentation of the wound tissue followed by extraction of the wound contour. The authors in ${ }^{[10]}$, used 50 RGB images, which were manually delineated by experts as training data, and then tested their method using 23 new RGB wound images. Their SVM algorithm was able to correctly classify roughly $94 \%$ of the pixels as either wound or non-wound, compared with the expert's manual tracings.

The objective of this study was to assess the progress of wound healing rate in foot wounds by means of texture analysis. Monitoring wound conditions over time is an important aspect of wound care, which nowadays is mainly carried out manually ${ }^{[2-7]}$. The development of a computer-based wound monitoring system will permit the definition of standard wound healing rates, minimize inter- and intra-observer variations, and permit the electronic storage and retrieval of this information as a standard part of the patient's medical record. Preliminary results of this study were also published ${ }^{[12]}$, where only 44 images in four different time points and a much smaller subset of texture features and geometrical measures were used for the analysis and the evaluation of the proposed method. In this study, we perform automated segmentation of the wounds based on snakes while the initial contour is automatically positioned to the area of interest. We follow up the rate of wound healing by means of features and geometrical measures extracted from the automated segmented wound areas in consecutive patient visits.

\section{Materials \& methods}

\subsection{Acquisition of wound images}

A total of 77 color digital images from 11 different subjects with foot wounds were recorded (a task carried out by the co-author Michalis Polyviou, Podiatrist) and they were followed up every third day for 21 consecutive days. In each visit the wound was examined, and appropriate treatment was given. All subjects were between 15 and 60 years of age, and had an ankle or foot wound with a minimum surface area of $1 \mathrm{~cm}^{2}$. All subjects received standard wound care between visits, which included bi-daily debridement, treatment with moist wound healing protocols, offloading when appropriate, and in some cases, active wound healing agents such as topical hydrogels, growth factors, and hyperbaric oxygen. Upon arrival to their scheduled appointments, wound photographs were taken before wound debridement was applied. 
All wound images were taken, with a commercially available 8-bit Nikon D100 digital SLR camera with Canfield Twin flash illumination and polarizing filters (Nikon D100: Melville, NY) and were revealing optimal visualization of the wound. The polarized filters reduce light reflection from the wound surface and allow a better estimation of wound boundary. Images were taken in rooms with the lights shut off and the objects (wounds) were only illuminated by the flash light on the camera. Previous studies ${ }^{[13]}$ have shown that the built-in flash on the camera is by itself able to provide adequate illumination for surface wound images. Picture resolution was 538×695 pixels. All pictures were saved as JPEG files and ruler stickers placed in the imaging plane of the wounds were used as size references. A written informed consent was obtained from each patient according to the instructions of the local ethics committee.

\subsection{I mage intensity normalization}

In this study, brightness adjustments of wound images were carried out based on the method introduced ${ }^{[2]}$. The method improves image compatibility by reducing the variability introduced by different gain settings, different operators, different equipment, and facilitates image tissue comparability. The intensity normalization process was performed on the grayscale image (see Figure 1b), which represents the first channel (luminance channel) of the original color wound image as follows:

$$
N(i, j)=\left(I(i, j)-G_{\min }\right) /\left(G_{\max }-G_{\min }\right)
$$

where $N(i, j)$ and $I(i, j)$ are the normalized and the original wound images respectively and $G_{\max }$ and $G_{\min }$ are the maximum and the minimum grayscale values in the original grayscale wound image respectively ${ }^{[2]}$. The values $G_{\max }$, $G_{\min }$ were manually selected by the user of the system. Thus, the original image histogram was stretched and shifted, in order to cover all the gray scale levels in the image. The resulting wound normalized image is shown in Figure 1c. The scale of the gray level of the images ranged from 0-255 and thus the brightness of all pixels in the image was readjusted according to the linear scale defined, by selecting the two reference regions $\left(G_{\max }, G_{\min }\right)$.

The above image normalization procedure, using the minimum and maximum grayscale values as reference points was necessary in order to extract comparable measurements in case of processing images obtained by different operators or different equipment at different time points. The image normalization procedure performed in this study was implemented in MATLAB software (version 7.9.0.529 (R2009b)), release 12 August, 2009, by The Mathworks, Inc.), and tested on a Pentium III desktop computer, running at $3.0 \mathrm{GHz}$, with 4.0 GB of RAM memory. The same software and computer station were also used for all other methods employed in this study.

\subsection{I mage preprocessing}

A wound initialization procedure was carried out for the purpose of positioning the initial snake contour as close as possible to the area of interest. The procedure is described as follows (see Figure 1): 1) Load the original color wound image (see Fig. 1a). 2) Compute and select the luminance channel of the image, which represent the grayscale image (see Figure 1b). 3) Perform image intensity normalization (see section 2.2). The normalized grayscale wound image is shown in Figure 1c. 4) Binarise the image by thresholding in order to extract edges more easily (see Figure 1d). A threshold is calculated from the normalized grayscale image ${ }^{[14]}$ so that the intraclass variance of the thresholded black and white pixels is minimized. 5) Dilate the binary image (from step 4) by a $3 \times 3$ pixel-structuring element consisting of ones, which is multiplied with the binary image (see Figure 1d). The pixel-structuring element represents a white mask with grayscale 255 and a size of $3 \times 3$ pixels. This morphological dilation operation is performed to close small gaps and to form a continuous boundary. 6) Remove erroneous small edges that might trap the snake segmentation algorithm (see section 2.4). This is carried out by labeling connecting components in the image where the number of connecting components in a pixel neighborhood was chosen to be eight. Small segments that are smaller than 20 pixels, and do not belong in the boundary are therefore removed. 7) Extract the contour matrix of the above area by locating points and their coordinates on wound boundaries, and construct an interpolating B-spline. Sample the interpolating B-spline, in equal distance points, in 
order to define a number of snake elements on the contour. The number of snake points is variable and is determined according to the area chosen by the user. The number of snake points is estimated by dividing the number of pixels belonging to the largest size of the area defined in Figure 1d, by 20. This will affect that fewer or more snake points will be defined for a smaller or a larger area respectively. 8) Map the detected contour points from step 7, on the normalized wound image of Fig. 1c, to form the initial snake contour for the wound. 9) Apply the snake segmentation algorithm ${ }^{\text {[15] }}$ (see also section 2.4) to extract and refine the final snake contours for the wound (see Figure 1f). 10) Extract the area of the wound (see Figure 1g) and draw its histogram (see Figure 1h).

\subsection{Snake segmentation}

The Williams \& Shah snake segmentation method ${ }^{[15]}$ was used to deform the snake and segment the final wound borders as follows:

$$
E_{\text {snake }}(v(s))=E_{\text {int }}(v(s))+E_{\text {image }}(v(s))+E_{\text {ext }}(v(s))=\int_{s}\left(\alpha(s) E_{\text {cont }}+\beta(s) E_{\text {curv }}+\gamma(s) E_{\text {image }}+E_{\text {external }}\right) d s
$$

with $E_{\text {int }}(v)$ and $E_{\text {ext }}(v)$ the internal and the external energies of the snake. An additional image energy term, $E_{\text {image }}(v)$, which is given by the negative gradient of the current contour point is proposed ${ }^{[16]}$. For the calculation of the snake parameters, $\alpha(s)$, and $\beta(s)$, we have empirically chosen in our study the initial values of $\alpha_{i}(s)=0.6, \beta_{i}(s)=0.4$, and $\gamma_{i}(s)=2$, to start the snake deformation. These values are consistent with other studies ${ }^{[15,16]}$. The extracted final snake contours are shown in Figure 1f (see also Figure 2c). The proposed snakes segmentation method was proposed and evaluated ${ }^{[16]}$, using 80 ultrasound images of the common carotid artery (CCA) and more details about the model can be found there. The automated segmented wound boundaries were also visually assessed by a doctor, who confirmed the correctness segmentation of the wounds.

\subsection{Texture features and geometrical measures}

In this study, 56 texture features and 4 geometrical measures were extracted from all segmented wounds. The overall texture features and geometrical measures for each subject were then estimated by averaging the corresponding values for all wounds for each subject. The following features and measures were extracted:

(1) Statistical Features (SF) ${ }^{[16,17]}$ 1) mean, 2) variance, 3) median value, 4) skewness, and 5) kurtosis.

(2) Spatial Gray Level Dependence Matrices (SGLDM) as proposed by Haralick et al. ${ }^{[17]}: 1$ ) angular second moment (ASM), 2) contrast, 3) correlation, 4) sum of squares variance (SOSV), 5) inverse difference moment (IDM), 6) sum average (SA), 7) sum variance (SV), 8) sum entropy (SE), 9) entropy, 10) difference variance (DV), 11) difference entropy (DE) and 12) information measures of correlation (IMC). For a chosen distance $d$ (in this work $d=1$ was used) and for angles $\theta=0^{\circ}, 45^{\circ}, 90^{\circ}$, and $135^{\circ}$, we computed four values for each of the above texture features. Each feature was computed by using a distance of one pixel. Then for each feature, the mean value and the range of values were computed, and were used as two different feature sets.

(3) Gray Level Difference Statistics (GLDS) ${ }^{[18]}$ : 1) contrast, 2) angular second moment, 3) entropy and 4) mean. The above features were calculated for displacements $\delta=(0,1),(1,1),(1,0),(1,-1)$, where $\delta \equiv(\Delta x, \Delta y)$, and their mean values were taken.

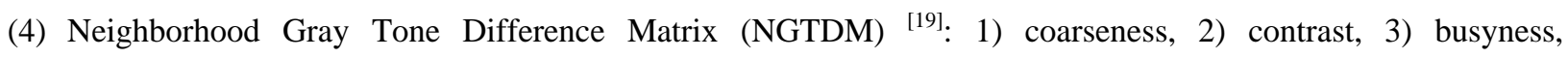
4) complexity, and 5) strength. 
(5) Statistical Feature Matrix (SFM) ${ }^{[20]}$ : 1) coarseness, 2) contrast, 3) periodicity, and 4) roughness.

(6) Laws Texture Energy Measures (LTEM) ${ }^{[20]}$ : 1) LL-texture energy from LL kernel, 2) EE-texture energy from EE-kernel, 3) SS-texture energy from SS-kernel, 4) LE-average texture energy from LE and EL kernels, 5) ES-average texture energy from ES and SE kernels, and 6) LS-average texture energy from LS and SL kernels.

(7) Fractal Dimension Texture Analysis (FDTA) ${ }^{[20]}$ : The Hurst coefficients $H^{(k)}$ for different dimensions k=2, 3, and 4 were computed. Each feature was computed using a distance of one pixel.

(8) Fourier Power Spectrum (FPS) ${ }^{[20]}$ : 1) radial sum, and 2) angular sum.

(9) Geometrical measures: 1) area, 2) perimeter, 3) X-coordinate maximum length, and 4) Y-coordinate maximum length, 5) perimeter2/area, 6) eccentricity, 7) equivalence diameter, 8) major axis length, 9) minor axis length, 10) centroid, 11) convex area, and 12) orientation.

\subsection{Statistical analysis}

As data were not normally distributed or had unequal variances, the Mann-Whitney rank sum test (for paired samples of different sizes) ${ }^{[21]}$, which calculates the difference between the sum of the ranks of two different independent samples was used in order to identify if for each texture feature and geometrical measure a significant difference (S) or not (NS) exists. The test was performed between the texture features and geometrical measures extracted from the wound images at all different visits with a confidence level of $95 \%$. For significant differences, we require a significance level, $p<0.05$. The test was implemented using the MATLAB ${ }^{\circledR}$ ranksum (x, y), which performs a two-sided rank sum test of the null hypothesis that data in the vectors $\mathrm{x}$ and $\mathrm{y}$ are independent samples from identical continuous distributions with equal medians, against the alternative that they do not have equal medians. The two data vectors $\mathrm{x}$ and $\mathrm{y}$ can have different lengths. In this study our data had unequal lengths as in the first visit we had 11 different samples (from 11 different subjects), while in the following visits (mainly in the 5th to 7th) we had a smaller size of samples because some of the subjects did not come to their appointments. Only those features that showed significant difference are presented in the results.

\section{Experimental results}

Figure 2 illustrates an example of a wound on a foot, which shows the progression of wound healing in seven consecutive patient visits (see 1st column in Figure 2). In the second column of Figure 2 we present the grayscale normalized image (see subsection II.B) of the wound for the seven different visit points of the patient, while in the third column we provide the automated wound segmentations (see subsections 2.3 and 2.4). In the fourth and fifth columns of Figure 2, we present the extracted wound areas after the automated segmentation by snakes and their respective histograms for all seven different patient visits respectively. It is evident that the area of the wound reduces at each visit (see Figure 2, 3rd column), and the histogram of the lesion (see Figure 2, 5th column) presents a different gray level distribution for the wound.

Table 1 illustrates the (mean values \pm standard deviation) for texture features that showed significant differences between consecutive visits, extracted from all wound areas of all 11 subjects for following up the progression of the wound healing rate. Only significantly different features are shown in Table 1 at $p<0.05$. Bolded values indicate significantly different features at a specific subjects visit. The statistical features are grouped under the categories SF, SGLDM range of values, GLDS, NGTDM, LTEM, FDTA and FPS. The Mann-Whitney rank sum test at $p<0.05$, was performed between consecutive visits in order to estimate significant differences between texture features. Some texture features increase (mean, contrast from the SF features group), while some other texture features decrease (entropy, SSV, SA, SV from the SGLDM range of values features group) with the progression of the wound healing. In the GLDS and the NGTDM feature groups we found that entropy and coarseness respectively decrease with the progression of the wound healing process. We found that the texture feature roughness from the SFM features group and the EE-kernel from the LTEM group decrease 
Table 1. Mean values \pm standard deviation for all wound texture features that showed statistical significant difference between consecutive visits at $p<0.05$.

\begin{tabular}{|c|c|c|c|c|c|c|c|}
\hline & Visit 1 & Visit 2 & Visit 3 & Visit 4 & Visit 4 & Visit 6 & Visit 7 \\
\hline \multicolumn{8}{|c|}{ SF-Statistical Features } \\
\hline Mean & $195 \pm 22$ & $\begin{array}{l}213 \pm 12 \\
(0.24)\end{array}$ & $\begin{array}{l}221 \pm 10 \\
(0.37)\end{array}$ & $\begin{array}{l}225 \pm 5 \\
(\mathbf{0 . 0 3 )}\end{array}$ & $\begin{array}{l}226 \pm 4 \\
(0.46)\end{array}$ & $\begin{array}{l}227 \pm 4 \\
(0.33)\end{array}$ & $\begin{array}{l}227 \pm 5 \\
(0.51)\end{array}$ \\
\hline Contrast & $90 \pm 40$ & $\begin{array}{l}120 \pm 50 \\
(0.29)\end{array}$ & $\begin{array}{l}160 \pm 80 \\
(0.77)\end{array}$ & $\begin{array}{l}160 \pm 50 \\
\mathbf{( 0 . 0 2 )}\end{array}$ & $\begin{array}{l}170 \pm 50 \\
(0.22)\end{array}$ & $\begin{array}{l}180 \pm 10 \\
(0.40)\end{array}$ & $\begin{array}{l}200 \pm 8 \\
(0.55)\end{array}$ \\
\hline \multicolumn{8}{|c|}{ SGLDM range of values-Spatial Gray Level Dependence Matrix } \\
\hline SSV & $0.81 \pm 0.12$ & $\begin{array}{l}0.77 \pm 0.12 \\
\mathbf{( 0 . 0 1 )}\end{array}$ & $\begin{array}{l}0.71 \pm 0.15 \\
(0.04)\end{array}$ & $\begin{array}{l}0.69 \pm 0.12 \\
(0.34)\end{array}$ & $\begin{array}{l}0.69 \pm 0.11 \\
(0.53)\end{array}$ & $\begin{array}{l}0.69 \pm 0.12 \\
(0.62)\end{array}$ & $\begin{array}{l}0.69 \pm 0.12 \\
(0.62)\end{array}$ \\
\hline $\begin{array}{l}\text { Sum } \\
\text { Variance }\end{array}$ & $449 \pm 14$ & $\begin{array}{l}434 \pm 15 \\
(0.29)\end{array}$ & $\begin{array}{l}418 \pm 26 \\
(0.32)\end{array}$ & $\begin{array}{l}401 \pm 43 \\
(0.87)\end{array}$ & $\begin{array}{l}395 \pm 43 \\
(0.93)\end{array}$ & $\begin{array}{l}367 \pm 23 \\
(0.38)\end{array}$ & $\begin{array}{l}344 \pm 91 \\
\mathbf{( 0 . 0 2 )}\end{array}$ \\
\hline $\begin{array}{l}\text { Sum } \\
\text { Average }\end{array}$ & $0.07 \pm 0.03$ & $\begin{array}{l}0.06 \pm 0.03 \\
\mathbf{( 0 . 0 2 )}\end{array}$ & $\begin{array}{l}0.05 \pm 0.03 \\
(0.79)\end{array}$ & $\begin{array}{l}0.04 \pm 0.03 \\
(0.63)\end{array}$ & $\begin{array}{l}0.04 \pm 0.03 \\
(0.32)\end{array}$ & $\begin{array}{l}0.04 \pm 0.03 \\
(0.38)\end{array}$ & $\begin{array}{l}0.04 \pm 0.03 \\
(0.76)\end{array}$ \\
\hline \multicolumn{8}{|c|}{ GLDS-Gray level Difference Statistics } \\
\hline Entropy & $5.2 \pm 0.3$ & $\begin{array}{l}5.1 \pm 0.4 \\
\mathbf{( 0 . 0 4 )}\end{array}$ & $\begin{array}{l}4.9 \pm 0.3 \\
\mathbf{( 0 . 0 3 )}\end{array}$ & $\begin{array}{l}4.8 \pm 0.2 \\
\mathbf{( 0 . 0 1 )}\end{array}$ & $\begin{array}{l}4.8 \pm 0.2 \\
(0.89)\end{array}$ & $\begin{array}{l}4.7 \pm 0.2 \\
(0.73)\end{array}$ & $\begin{array}{l}4.6 \pm 0.2 \\
(0.62)\end{array}$ \\
\hline \multicolumn{8}{|c|}{ NGTDM-Neighborhood Gray Tone Difference Matrix } \\
\hline Coarseness & $154899 \pm \mathrm{E} 10$ & $\begin{array}{l}126960 \pm \mathrm{E} 10 \\
(0.34)\end{array}$ & $\begin{array}{l}\text { 108582+E9 } \\
\mathbf{( 0 . 0 1 5 )}\end{array}$ & $\begin{array}{l}75260 \pm E 7 \\
(0.87)\end{array}$ & $\begin{array}{l}11006 \pm E 6 \\
(0.47)\end{array}$ & $\begin{array}{l}66849 \pm \mathrm{E} 6 \\
(0.7)\end{array}$ & $\begin{array}{l}78252 \pm \mathrm{E} 5 \\
(0.82)\end{array}$ \\
\hline \multicolumn{8}{|c|}{ SFM-Statistical Feature Matrix } \\
\hline Roughness & $0.48 \pm 0.05$ & $\begin{array}{l}0.51 \pm 0.05 \\
\mathbf{( 0 . 0 0 7 )}\end{array}$ & $\begin{array}{l}0.53 \pm 0.05 \\
\mathbf{( 0 . 0 4 )}\end{array}$ & $\begin{array}{l}0.56 \pm 0.05 \\
(0.96)\end{array}$ & $\begin{array}{l}0.56 \pm 0.05 \\
(0.91)\end{array}$ & $\begin{array}{l}0.56 \pm 0.05 \\
(0.82)\end{array}$ & $\begin{array}{l}0.57 \pm 0.05 \\
(0.91)\end{array}$ \\
\hline \multicolumn{8}{|c|}{ LTEM-Laws Texture Energy Measures } \\
\hline $\mathrm{EE}$ & $87810 \pm 3 E 3$ & $\begin{array}{l}72512 \pm 2 E 3 \\
(0.4)\end{array}$ & $\begin{array}{l}52920 \pm 2 E 3 \\
\mathbf{( 0 . 0 0 7 )}\end{array}$ & 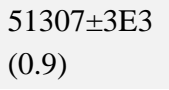 & $\begin{array}{l}55067 \pm 8 E 3 \\
(0.68)\end{array}$ & $\begin{array}{l}49446 \pm 3 E 3 \\
(0.94)\end{array}$ & $\begin{array}{l}37438 \pm 3 E 3 \\
(0.7)\end{array}$ \\
\hline \multicolumn{8}{|c|}{ FDTA-Fractal Dimension Texture Analysis } \\
\hline $\mathrm{H} 2$ & $0.36 \pm 0.56$ & $\begin{array}{l}0.35 \pm 0.05 \\
(0.31)\end{array}$ & $\begin{array}{l}0.33 \pm 0.05 \\
\mathbf{( 0 . 0 3 )}\end{array}$ & $\begin{array}{l}0.32 \pm 0.05 \\
(\mathbf{0 . 0 5})\end{array}$ & $\begin{array}{l}0.32 \pm 0.03 \\
(0.48)\end{array}$ & $\begin{array}{l}0.32 \pm 0.04 \\
(0.77)\end{array}$ & $\begin{array}{l}0.32 \pm 0.03 \\
(0.76)\end{array}$ \\
\hline H4 & $0.30 \pm 0.09$ & $\begin{array}{l}0.27 \pm 0.09 \\
(0.29)\end{array}$ & $\begin{array}{l}0.22 \pm 0.1 \\
\mathbf{( 0 . 0 4 )}\end{array}$ & $\begin{array}{l}0.23 \pm 0.09 \\
(0.9)\end{array}$ & $\begin{array}{l}0.23 \pm 0.05 \\
(0.73)\end{array}$ & $\begin{array}{l}0.23 \pm 0.03 \\
(0.76)\end{array}$ & $\begin{array}{l}0.23 \pm 0.07 \\
(0.55)\end{array}$ \\
\hline \multicolumn{8}{|c|}{ FPS-Fourier Power Spectrum } \\
\hline Radial Sum & $0.07 \pm 0.08$ & $\begin{array}{l}0.13 \pm 0.07 \\
(0.4)\end{array}$ & $\begin{array}{l}0.18 \pm 0.06 \\
\mathbf{( 0 . 0 3 )}\end{array}$ & $\begin{array}{l}0.23 \pm 0.05 \\
(0.98)\end{array}$ & $\begin{array}{l}0.23 \pm 0.04 \\
(0.47)\end{array}$ & $\begin{array}{l}0.23 \pm 0.02 \\
(0.91)\end{array}$ & $\begin{array}{l}0.23 \pm 0.02 \\
(0.7)\end{array}$ \\
\hline
\end{tabular}

Note. SSV: Sum of squares variance, EE: EE-texture energy from EE-kernel, H2, H4: Hurst coefficients for dimensions 2 and 4. $P$-values are shown in parentheses. Bolded values indicate significantly different features at $p<0.05$.

The Mann-Whitney rank sum test performed in Table 1, showed different statistical significant differences for the texture features between consecutive visits. Texture features mean and contrast (see also Table $1, p=0.03$ and $p=0.02$ respectively) showed a significant difference after the 4 th visit, texture features SSV, SA and entropy ( $p=0.01, p=0.02$ and $p=0.04$ respectively) showed a significant difference after the 2 nd visit, while the texture feature sum variance $(p=0.02)$ showed a significant difference after the 7th visit. Coarseness and roughness indicated a significant difference after the 3rd and 2nd visit respectively ( $p=0.015$ and $p=0.07$ respectively), while EE, $\mathrm{H} 2$ and $\mathrm{H} 4$ indicated a significant difference after the 2nd visit ( $p=0.007, p=0.03$ and $p=0.04$ respectively). Finally, radial sum showed a significant difference after the 3rd week $(p=0.03)$.

Table 2 illustrates the (mean \pm standard deviation) of geometrical measures (in number of pixels) of, area, perimeter, $\mathrm{x}$-coordinate length and $\mathrm{y}$-coordinate length and their respective p-values between consecutive visits. These were extracted from all 11 subjects, from all wounds for seven consecutive visits in order to monitor the progression of the wound healing rate. It is observed that all four geometrical measures investigated in this study decrease with the 
progression of wound healing. The Mann-Whitney rank sum test performed between the geometrical measures for all different visits showed no significant differences between them, as observed by the p-values in Table 2 .

Table 2. Mean \pm standard deviation values for geometrical measures (in number of pixels) extracted from all wounds.

\begin{tabular}{llllllll}
\hline & Visit 1 & Visit 2 & Visit 3 & Visit 4 & Visit 5 & Visit 6 & Visit 7 \\
\hline \multirow{2}{*}{ Area } & $103 \pm 70$ & $87 \pm 51$ & $89 \pm 47$ & $81 \pm 39$ & $79 \pm 37$ & $78 \pm 13$ & $57 \pm 17$ \\
& $(0.71)$ & $(0.33)$ & $(0.44)$ & $(0.32)$ & $(0.69)$ & $(0.28)$ & $(0.22)$ \\
Perimeter & $10320 \pm 1071$ & $8890 \pm 8758$ & $5474 \pm 5325$ & $4399 \pm 3832$ & $8265 \pm 6523$ & $3801 \pm 2056$ & $2108 \pm 821$ \\
& $(0.43)$ & $(0.51)$ & $(0.34)$ & $(0.99)$ & $(0.78)$ & $(0.76)$ & $(0.44)$ \\
X-coordinate & $4750 \pm 3561$ & $4008 \pm 2650$ & $3803 \pm 2636$ & $3745 \pm 2071$ & $3328 \pm 2750$ & $2956 \pm 841$ & $2588 \pm 1595$ \\
& $(0.22)$ & $(0.11)$ & $(0.12)$ & $(0.22)$ & $(0.56)$ & $(0.67)$ & $(0.45)$ \\
Y-coordinate & $109 \pm 64$ & $95 \pm 50$ & $86 \pm 34$ & $78 \pm 31$ & $103 \pm 34$ & $75 \pm 25$ & $58 \pm 8$ \\
& $(0.33)$ & $(0.45)$ & $(0.11)$ & $(0.21)$ & $(0.77)$ & $(0.87)$ & $0.61)$ \\
\hline
\end{tabular}

Note. $P$-values are shown in parentheses.

Figure 3a) presents box plots for selected texture features, (mean (m) and sum variance (SV)), that showed significant differences through the wound healing process, while Figure 3b) illustrates the sum of squares variance (SSV) and the sum average (SA) for seven consecutive visits (W1, W2, W3, W4, W5, W6, W7). We observe that the texture feature median increase, while the texture features SV, SSV and SA decrease with the progression of wound healing.

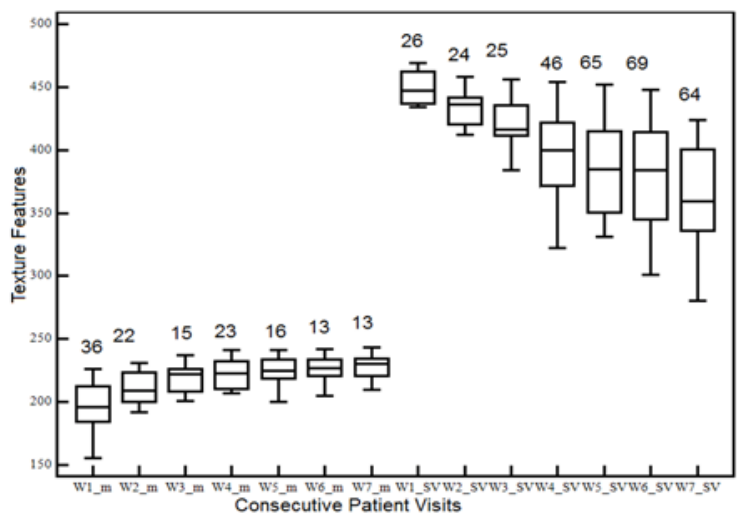

a)

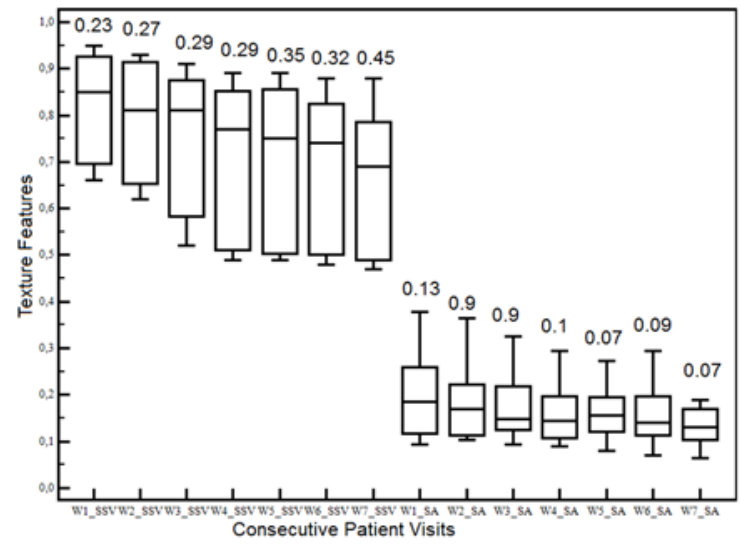

b)

Figure 3. Box plots for wound texture features from all subjects a) mean (m) and sum variance (SV), b) sum of squares variance (SSV) and sum average (SA) for seven consecutive patient visits (W1, W2, W3, W4, W5, W6, W7). In each plot we display the median, lower, and upper quartiles and confidence interval around the median. Straight lines connect the nearest observations within 1.5 of the IQR of the lower and upper quartiles. Unfilled triangles indicate possible outliers with values beyond the ends of the $1.5 \times$ IQR. 


\section{Discussion}

\subsection{General discussion}

The objective of this study was to establish texture features and geometrical measures extracted in consecutive patient visits from foot wounds that may describe the rate of wound healing. It is shown that it may be possible to follow up the progression of wound healing rate with the use of texture features extracted from the wound areas. We have studied 11 different subjects from which 77 color images with foot wounds were acquired every third day, for 21 days. The subjects suffered from wounds on their feet and were treated for seven consecutive time points (visits), while digital images of the wounds were taken before treatment at each visit. The wounds were intensity normalized and automatically segmented. Texture features and geometrical measures were extracted from the wounds in an attempt to find features that may describe the wound healing process. We found statistical significant differences between some of these features, as follows: 1) The features mean, contrast, roughness and radial sum, showed an increase, and 2) The features SSV, SV, SA, entropy, coarseness, $\mathrm{H} 2$ and H4, showed a decrease with the progression of wound healing (see also Table 1 and Fig. 3). Table 1 also shows that some texture features can be used to follow the progression of wound healing and it might be possible to estimate the time in which the wound heals. Table 2 shows that the geometrical measures extracted from the wounds are not significantly different between consecutive visits, thus are not useful to be used for indicating the wound healing rate but they may be used for wound measurements. Image analysis is becoming a powerful tool in the diagnosis of different wound conditions ${ }^{[2-8]}$. The proposed methodology is suitable for monitoring wound healing and the rate of healing.

We have not found other studies in the open literature where texture features extracted from wounds are used to monitor the progression of wound healing rate. There are some earlier studies by other researchers for measuring the wound size in order to follow the wound healing rate. Two measuring methods ${ }^{[22]}$, namely the acetate and planimetry are proposed against the manual measurement of wounds with the ruler. Accurate wound assessment and measurements are essential documentation parameters in the treatment of chronic and acute wounds. Changes in wound size and tissue type are key indicators in monitoring the healing progress of a wound ${ }^{[22]}$. Clinicians regularly face the complex tasks of choosing the appropriate treatment method and wound care product specific to the rate and the exact time point of healing.

Two statistical measures ${ }^{[13]}$, namely the kurtosis and the asymmetry, were extracted from 71 color images of burn wounds in order to classifying the deepness of the wound. They managed to classify the wounds with a classification success rate of $88.75 \%$. In another study the use of digital image processing using hue, saturation, and intensity measurements as a technique for the color analysis of chronic wounds on the skin was presented ${ }^{[23]}$. An adaptive spline technique was used to segment the wound boundary in the images of venous leg ulcers. The technique was further used to approximate the position of venous leg ulcers. The amount of slough within the wound site was quantified using a software developed and was compared with a grading system based on visual inspection by an experienced clinician, and the results were compared by deriving Kappa statistic ${ }^{[21]}$. There was moderate agreement over all grades between the computer and the clinician, while at lower grades, there was an excellent agreement. The results ${ }^{[23]}$ suggest that there is a potential to apply image processing techniques to follow the progression of chronic skin wounds. It was shown that an important clinical feature of skin cancer is the asymmetry of skin lesions where its highly desirable to identify malignant melanoma, a common cancer, at an early stage ${ }^{[24]}$. The geometrical characteristics extracted from the wounds in this research may also help in this direction.

There are also some other studies proposed in the literature, where automated systems were proposed for following up the wound healing process. More specifically, the authors proposed an imaging system useful in dermatology, for the follow-up of subjects with an increased risk of skin cancer. The system allowed colorimetrically consistent acquisition of images ${ }^{[25]}$. 
Flanagan ${ }^{[26]}$ suggested an advantage of wound size monitoring is that plotting healing rates against initial wound area and then comparing them with a defined standard helps to inform clinical decision-making and reduces the likelihood of ineffective treatments. Eventually, this information may help in establishing baseline healing rates for different wound types, which would facilitate meta-analysis, allow objective comparisons of different treatments and assist reliable cost-benefit analysis ${ }^{[26]}$. It was also reported that nurses often report on the type of tissue found in the wound and use this as an indicator of progress towards healing. The analysis of wound tissue is a key parameter required in understanding exactly what is happening within the wound bed and whether the current treatment method is improving the wound healing process $^{[26]}$.

\subsection{Study limitations}

The initialization of the contour as presented in this study, for estimating the initial borders of the wound, poses a certain limitation of the proposed method. If the initial contour for the wound is placed far away from the correct boundary then the snake contour will end up in a wrong location. Therefore, a more robust initialization method should be investigated in future. Another limitation of the method is that manual segmentations of the wounds were not necessarily performed by expert(s). In such a case, the automated segmented wound boundaries could be compared with the manual segmented boundaries and the method would be therefore correctly evaluated. Another limitation is the small number of images, in which the current method was applied. A further limitation is that all different types of wounds, of different initial size and of various tissue types are collectively treated. By treating them as a single group, individual differences are masked. This is evident in Table 2. We intent to address this issue appropriately when more images are acquired. We are currently collecting additional images and we intent to perform the study on a larger number of images in order to validate the results of this study.

\subsection{Future directions}

Further research work on a larger number of subjects, where subjects may also be grouped into different groups of ages is required for validating the results of this study and for finding additional texture features and geometrical measures that may provide information for following up the wound healing rate.

The research could also be extended to 3D imaging, as it is proposed, where an approach to build 3D models of skin wounds from color images was presented. It is claimed that from a 3D model of the skin wound accurate volumetric measurements could be achieved, with a global precision around 3\%. This is in accordance with a general clinical requirement of $5 \%$ for assessing the healing process ${ }^{[27]}$.

Improvements in the measurement and pre-processing of the images are expected to have a significant impact in enhancing both the clinical and the computer-aided assessment. The method of histogram-intensity normalization used in this study (see section 2.2) ${ }^{[2]}$ was a key step in obtaining consistent comparisons, however, other pre-processing methodologies could be investigated. This will enable a more accurate extraction of texture features that may provide additional information for better differentiation between normal and abnormal tissues and in assessing wound healing progress. The proposed method demonstrates a system suitable for following the wound healing rate.

The proposed methodology could be further developed and be integrated into a software application. Health care professionals, such as dermatologists, podiatrists, and primary care nurses who are involved in wound care and management in a hospital, clinic or in the homes of patients could benefit by a such application.

Pixel intensity variations between the same and consecutive wound images acquired at different consecutive visits and irregularity of the wound healing rate may complicate the method of quantitative wound analysis ${ }^{[2]}$. 


\subsection{Concluding remarks}

This paper presents a study on the evaluation of wound healing rate based on texture analysis. Wound healing rate is an interesting and important issue, in which modern imaging techniques have not yet given a definitive answer. Distorted wound healing can lead to a number of undesirable conditions such as non-healing of chronic wounds that may lead to infections, wounds that can become dangerously infected with a high risk of excessive inflammation. There is an increasing interest in the medical community to apply new innovative computer aided methods in order to guide better therapeutic interventions and improve understanding of the fundamental mechanisms driving tissue repair in wounds.

Our objective in this study was to investigate whether texture features and geometrical measures which, were extracted from wounds on foot at consecutive subjects visits, may describe the rate of wound healing. We have investigated color digital images of wounds on feet, taken from 11 subjects at seven different office visits. After image normalization, wounds were automatically segmented followed by texture features and geometrical measures extraction from the segmented wounds, in an attempt to describe the wound healing rate. We found statistical significant differences in some texture features, specifically, mean, contrast, roughness and radial sum, SSV, SV, SA, entropy, coarseness, H2 and H4. The geometrical measures showed no significant differences between consecutive visits. It is thus our conclusion that there are some texture features that may be useful for monitoring the degree of the wound healing rate. This will probably give an additional tool to the examining specialist for deciding on the correct treatment of the wound.

Further research work on a larger number of subjects is required for validating the results of this study and for finding additional texture features and geometrical measures that may provide information for longitudinal monitoring of the wound healing rate of subjects with wounds. The proposed system will be further evaluated on a larger sample of images as well as incorporate other texture features and measures which may quantify the rate of wound healing. The development of such a system will permit the definition of standard wound healing rates, minimize inter- and intra-observer variations, and permit the electronic storage and retrieval of this information as a standard part of the patient's medical record. We anticipate that the proposed system may be incorporate in the clinical practice in an attempt to assist health care professionals, such as dermatologists, podiatrists and nurses, for more accurate diagnosis and treatment of wounds. We also believe that the above study may have some clinical value in following the progression of the wound.

The proposed methodology may also be applied in the estimation of deep wounds, such as pressure ulcers, or deep tissue injuries to patient's tissue below the skin's surface, which is a major challenge. This kind of wounds has very small surface areas where color-based detection can lead to erroneous results and which could be overcome by texture-based detection methods.

\section{References}

[1] Boulton AJM, Vileikyte L, Ragnarson-Tennvall G, Apelqvist J. The global burden of diabetic foot disease. Lancet. 2005; 366: 1719-24. http://dx.doi.org/10.1016/S0140-6736(05)67698-2

[2] Papazoglou ES, Zubkov L, Mao X, Neidrauer M, Rannouy N, Weingarten MS. Image analsyis of chronic wounds for determining the surface area. Wound Rep Reg. 2010; 18: 349-58. PMid:20492631 http://dx.doi.org/10.1111/j.1524-475X.2010.00594.x

[3] Whitney J, Phillips L, Aslam R, Barbul A, Gottrup F, Gould L, et al. Guidelines for the treatment of pressure ulcers. Wound Rep Reg. 2006; 14: 663-79. PMid:17199832 http://dx.doi.org/10.1111/j.1524-475X.2006.00175.x

[4] Jones BF, Plassman P. An instrument to measure the dimensions of skin wounds. IEEE Trans Biomed Eng. 1995; $42(5): 464-70$. http://dx.doi.org/10.1109/10.376150

[5] Jones TD. Semi-automatic segmentation algorithms for measuring the area of skin wounds. University of Glamorgan, Department of Computer Studies. Computer Studies Technical Report. 1994; CS-94-3.

[6] Mekkes JR, Westerhof W. Image processing in the study of wound healing. Clinics Dermatol. 1995; 13(4): $401-07$. http://dx.doi.org/10.1016/0738-081X(95)00071-M

[7] Gammal el S, Popp R. A color image analysis system (cd-cwa) to quantify wound healing of ulcers. Skin Resear Techn. 1995; 1(3): 158. 
[8] Solomon C, Munro AR, van Rij AM, Christie R. The use of video image analysis for the measurement of venous ulcers. British J Dermatol. 1995; 133: 565-70. http://dx.doi.org/10.1111/j.1365-2133.1995.tb02706.x

[9] Smith DJ, Bhat S, Bulgrin JP. Video image analysis of wound repair. Wounds. 1992; 4(1): 6-15.

[10] Kolesnik M, Fexa A. Segmentation of wounds in the combined color-texture feature space. SPIE 2004: Proceedings of Medical Imaging; 2004 Feb 16-19; San-Diego, USA; 2004. p. 549-556.

[11] Vapnik V. The Nature of Statistical Learning Theory. Springer, New York; 1995. PMid:8555380

[12] Loizou CP, Kasparis T, Mitsi O, Poliviou M. Evaluation of wound healing process based on texture analysis. BIBE 2012: 12th International Conference on Bioinformatics \& Bioengineering; 2012 Nov 11-13; Larnaca, Cyprus; 2012. pages 4.

[13] Acha B, Serrano C, Acha JI, Roa LM. Segmentation and classification of burn images by color and texture information. J Biomed Opt. 2005; 10(034014): 1-11.

[14] Otsu N. A Threshold Selection Method from Gray-Level Histograms. IEEE Trans Syst Man Cy. 1979; 1(9): 62-6.

[15] Williams DJ, Shah M. A fast algorithm for active contours and curvature estimation. Int J Graph Vision and Imag Proc: Image Underst. 1992; 55:14-26.

[16] Loizou CP, Pattichis CS, Pantziaris M, Nicolaides AN. An integrated system for the segmentation of atherosclerotic carotid plaque. IEEE Trans Inform Techn Biomed. 2007 Nov; 11(6): 661-7. PMid:18046941 http://dx.doi.org/10.1109/TITB.2006.890019

[17] Haralick RM, Shanmugam K, Dinstein I. Texture features for image classification. IEEE Trans Systems, Man, and Cybernetics. 1973 Nov; SMC-3:610-21. http://dx.doi.org/10.1109/TSMC.1973.4309314

[18] Weszka JS, Dyer CR, Rosenfield A. A comparative study of texture measures for terrain classification. IEEE Trans Syst, Man Cybern. 1976; SMC 6: 269-85.

[19] Amadasun M, King R. Textural features corresponding to textural properties. IEEE Trans. Syst., Man. Cybern. 1989 Sep; 19(5): 1264-74. http://dx.doi.org/10.1109/21.44046

[20] Wu CM, Chen YC, Hsieh K-S. Texture features for classification of ultrasonic images. IEEE Trans Med Imag. 1992 June; 11: 14152. PMid:18218367 http://dx.doi.org/10.1109/42.141636

[21] Bland JM, D. Altman G. Statistical methods for assessing agreement between two methods of clinical measurement. Lancet. 1986; 1(8476): 307-10. http://dx.doi.org/10.1016/S0140-6736(86)90837-8

[22] Gethin G. The importance of contuinous wound measuring. Wounds. 2006; 2(2): 60-8.

[23] Oduncu H, Hoppe A, Clark M, Williams R J, Harding K G. Analysis of skin wound images using digital color image processing: a preliminary communication. Int J Low Extrem Wounds. 2004; 3(3): 151-56. PMid:15866806 http://dx.doi.org/10.1177/1534734604268842

[24] Ng VT, Fung BY, Lee TK. Determining the asymmetry of skin lesion with fuzzy borders. Comp Biol Med. 2005; 35(2): 103-20. PMid:15567181 http://dx.doi.org/10.1016/j.compbiomed.2003.11.004

[25] Haeghen YV, Naeyaert JM, LemahieuI, PhilipsW. An imaging system with calibrated color image acquisition for use in dermatology. IEEE Trans Med Imag. 2000; 19(7): 722-30. PMid:11055787 http://dx.doi.org/10.1109/42.875195

[26] Flanagan M. Wound measurement: can it help us to monitor progression to healing? J Wound Care. 2003; 12(5): 189-94. PMid:12784601

[27] Treuillet S, Albouy B, Lucas Y. Three-dimensional assessment of skin wounds using a standard digital camera. IEEE Trans Med Imag. 2009; 28(5): 752-62. PMid:19150787 http://dx.doi.org/10.1109/TMI.2008.2012025 